\title{
High Throughput Laser Drilling with High Power Lasers Using a Two-Dimensional Polygon Mirror Scanner
}

\author{
Florian Rößler ${ }^{* 1}$, Mathias Müller ${ }^{2}$, and André Streek ${ }^{1,2}$ \\ ${ }^{1}$ MOEWE Optical Solutions GmbH, Schillerstr. 10, 09648 Mittweida, Germany \\ ${ }^{2}$ Laserinstitut Hochschule Mittweida, Technikumplatz 17,09648 Mittweida, Germany \\ *Corresponding author's e-mail: roessler@moewe-optik.de
}

\begin{abstract}
The throughput of laser processes is typically limited by the available average laser power or the distribution speed of the laser energy onto the workpiece. In the last years, the development of laser sources brought out high average power laser sources. Thus, the distribution speed becomes the limiting factor. The development of industry ready polygon mirror scanners addresses this problem. In this study, a $1 \mathrm{~kW}$ nanosecond pulsed fiber laser is used to drill silicon wafers of $180 \mu \mathrm{m}$ thickness and stainless-steel of $200 \mu \mathrm{m}$ thickness in multi pass ablation. The laser energy is distributed with a two-dimensional polygon mirror scanner with a free aperture of $30 \mathrm{~mm}$. Scan speeds up to $400 \mathrm{~m} / \mathrm{s}$ has been utilized with high precision. In the used process, every drilling hole is hit only once per scan and must be hit again in every repetition until the holes is finished. This is achieved by pulse synchronization, whereby the laser adapts its pulse repetition rate to the scanner generated position frequency signal. In different processing conditions, between 25 and 220 pulses are required to drill through. With pulse repetition rates in the $\mathrm{MHz}$ range, drill rates of up to 15,000 holes/s are achievable. The accuracy of the process is evaluated using the drilling hole diameter at the laser entrance and exit side of the material. Furthermore, it is shown, how the diameter is influenced by variable processing parameters
\end{abstract}

DOI: $10.2961 / \mathrm{jlmn} .2020 .03 .2011$

Keywords: polygon mirror scanner, laser drilling, high throughput, high laser power, high accuracy

\section{Introduction}

Increasing throughput in laser processing is typically associated with an increase of the utilized average laser power. Modern pulsed laser sources can provide several kilowatts average power together with high pulse repetition rates in the $\mathrm{MHz}$-range. In cases of multi pulse treatments as in percussion drilling, the high pulse frequency can lead to limitations of the process efficiency until impracticability. Especially, heat accumulation can destroy the bore hole geometry by unwanted melting of material. Such thermal damage has been reported during ultra-short pulse laser treatments working in the so called "cold ablation" regime and will occur even more on longer pulse durations [1-4]. The heat accumulation strongly depends on the pulse repetition rate as several models has been shown $[1,4]$. The remaining heat in the bore hole cannot dissipate into the bulk material before the next pulse arrive. To keep the temperature in the borehole below the melting point of $\mathrm{Cr} / \mathrm{Ni}$ steel $\left(1,500{ }^{\circ} \mathrm{C}\right)$, an average laser power of $3.2 \mathrm{~W}$ must not be exceeded using al $\mathrm{MHz}$ pulse repetition rate [1]. Indeed, a decreased number of pulses to drill through is reported during a slightly increase of temperature due to heat accumulation with very low pulse energies [3]. Nevertheless, power limitations to avoid thermal damage do not enable a high throughput processing. Furthermore, above $500 \mathrm{kHz}$ shielding effects of consecutive pulses reduce the absorption in the target material due to particles from the previous laser pulse $[2,3]$.

A possibility to overcome the heat accumulation and pulse-to-pulse shielding during multi pulse drilling is found in multi-pass processing. Consecutive laser pulses are distributed on the whole target area by fast beam deflection. The required number of pulses to drill through is achieved by a correlating number of scans. Thus, the high pulse repetition rate respectively the high average power can be used while a single bore hole is treated with a significantly reduced frequency. This kind of processing requires a very high accuracy of positioning, to meet the ablated areas precise in every repetition. Using this method, low beam deflection speeds can limit the process. Between two consecutive laser pulses, the beam has to be deflected by the spacing distance of two neighbored holes. Since the beam deflection speed of galvo scanners is limited to some $10 \mathrm{~m} / \mathrm{s}$ with working moderate focal length below $500 \mathrm{~mm}$, a scanned distance of only several $10 \mu \mathrm{m}$ is achieved at a pulse frequency of $1 \mathrm{MHz}$ resulting in pulse overlap and not pulse separation [5].

The development of polygon scanners addresses this problem. Besides self-made solutions, a couple of commercial products are in the market now and make ultra-fast laser beam deflection capable for a wide range of users [6,7]. The unique combination of a scan speed up to $1,000 \mathrm{~m} / \mathrm{s}$, obtained with a $420 \mathrm{~mm}$ focal length objective, together with multiple kilowatt average power lasers is able to increase the throughput of laser processes significantly [8].

Laser drilling is a process, which can be speed up with the polygon scanning technology and is at the same time a process requiring high accuracy for laser pulse positioning. Laser drilling is already used in industry and research for a long time to fabricate for example micro holes in fluidic elements, such as nozzles or filters [9-11]. Drilled stainless- 
steel can be used for fine filtering [12]. Silicon is a material, which is widely used in the fabrication of micro electro mechanical systems or photovoltaics [13,14]. A demand on high throughput drilling is also reported from aerospace research for nickel and titanium alloys expecting more than 100,000 holes in future aero engines $[15,16]$. For example, a drill rate of 400 holes/s was achieved with a $200 \mathrm{~W}$ nspulsed fiber laser in $800 \mu \mathrm{m}$ thick titanium alloy [16].

This study investigates, how high average laser power of $1 \mathrm{~kW}$, obtained from pulse repetition rates of $1-4 \mathrm{MHz}$ and high pulse energy up tol $\mathrm{mJ}$, can be combined with scan speed of $100-400 \mathrm{~m} / \mathrm{s}$ to obtain high throughput, respectively high drilling rates. Thereby, the achieved bore hole diameters are analyzed. The effective pulse rate of a single bore hole, can be estimated from the pulse repetition rate and the size of the scanned area.

\section{Experimental}

The drilling experiments were performed on polycrystalline silicon wafers with a thickness of $180 \mu \mathrm{m}$ and stainlesssteel substrates of $200 \mu \mathrm{m}$ thickness. As laser source, an IPG fiber laser with a maximum average laser power of $1000 \mathrm{~W}$ and a wavelength of $1064 \mathrm{~nm}$ was used. The pulse repetition rate can be set to 1-4 MHz, depending on the pulse duration, which was varied between 30,60 and 240 ns. To distribute the laser energy on the surface, a polygon mirror scanner (PM-series from MOEWE Optical Solutions $\mathrm{GmbH}$, Mittweida, Germany) was used in all cases with an $\mathrm{f}-\theta-\mathrm{ob}-$ jective (from Sill optics $\mathrm{GmbH} \&$ Co. KG, Germany) of $420 \mathrm{~mm}$ focal length. An overview of the optical set up is visible in Fig. 1.

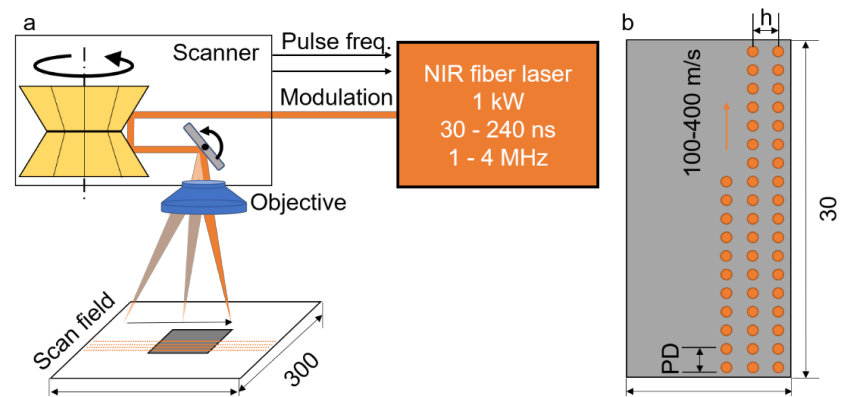

Fig. 1 (a) Experimental setup with $1 \mathrm{~kW}$ ns-pulsed NIR fiber laser and a $2 \mathrm{D}$ polygon mirror scanner with a $300 \times 300 \mathrm{~mm}^{2}$ scan field, (b) laser pulse distribution during multi-pass ablation drilling.

The laser beam enters the scanner, where a fast-rotating retroreflecting polygon mirror with eight facets reflects the light twice. The mechanical angular velocity of this mirror $\omega$ is transposed into a doubled optical angular velocity $\dot{\theta}$ following $\dot{\theta}=2 \omega=4 \pi \cdot \mathrm{rpm}$, with $\mathrm{rpm}$ as revolutions per minute. Furthermore, the angular velocity is transposed by the $\mathrm{f}-\theta$-objective into the scan speed $v_{\text {scan }}$ described by $v_{\text {scan }}=\dot{\theta} \cdot f$, with the focal length $f$.

The double reflection on the polygon mirror has two significant advantages compared to a flat mirror. Firstly, a back reflection in the incoming direction is avoided. Secondly, both $90^{\circ}$ reflections on the polygon mirrors compensate the each other's pivot point error occurring from the different radii at the edges and in the middle of a facet. Consequently, a virtual pivot point is created, which has neglectable fluctuations compared to other polygon mirror geometries.
After the polygon mirror, the laser beam is deflected once more at a galvanometer mirror. This is used to reflect the laser in the second direction (perpendicular to the line scan) and allows a displacement of the scanned lines to treat an area. At the scanner exit, the f- $\theta$-objective focusses the laser beam. The resulting scan field is $300 \times 300 \mathrm{~mm}^{2}$. Due to the fast and continuous rotation, the laser beam is scanned along a line with a constant scan speed.

At the end of a facet, the beam is switched off until the next facet is in position and the consecutive line is scanned. This break is called facet exchange gap. However, if the machined area is smaller than the length of a scanned line, the laser is switched off earlier. The target area in the experiments is set to $30 \mathrm{~mm}$ length and $5 \mathrm{~mm}$ width.

All experiments were performed with multi-pass ablation, where the beam is deflected continuously and the consecutive laser pulses arrive the work piece on different positions. In Fig. 1(b) it is visible, how the consecutive laser pulses were distributed over the target area. Under each condition of pulse duration and repetition rate, several treatments were performed slightly increasing the pulse number per hole in 5 or 10 pulse steps until a drill through can be observed.

To hit the same position in every scan repetition, a synchronization of the rotation frequency of the polygon and the pulse repetition rate must be enabled. The used laser device is able to adapt the pulse repetition rate to an external signal, which was provided by the polygon mirror scanner and varies between 1 and $4 \mathrm{MHz}$. Thereby, the signal is basically generated from the number of scanned positions per second concerning a 24-bit resolution of the facet. Hence, the signal respects the scan speed as well as fluctuations of it. To achieve a spacing of 100 or $200 \mu \mathrm{m}$ between the drilling holes, the scan speed was set to $100-400 \mathrm{~m} / \mathrm{s}$.

The diameters of the holes were analyzed using an optical microscope.

\section{Results and discussion}

Stainless-steel of $200 \mu \mathrm{m}$ thickness and polycrystal silicon wafers of $180 \mu \mathrm{m}$ thickness were treated with $1 \mathrm{~kW}$ average laser power, which is the maximum available power of the used laser source. This power can be obtained at pulse repetition rates from $1 \mathrm{MHz}$ to $4 \mathrm{MHz}$, with decreasing pulse energy from $1 \mathrm{~mJ}$ to $0.25 \mathrm{~mJ}$, respectively. However, increasing pulse repetition rates are only available with decreasing pulse duration. Using the full power of $1 \mathrm{~kW}$ with a frequency of $4 \mathrm{MHz}$ and the pulse duration is $30 \mathrm{~ns}$, the notation is $4 \mathrm{MHz} @ 30 \mathrm{~ns}$. Full power at $1 \mathrm{MHz}$ is available only with $240 \mathrm{~ns}$ pulse duration. Pulses of $60 \mathrm{~ns}$ can be obtained at $2 \mathrm{MHz}$ or $4 \mathrm{MHz}$. The pulse energy results from the average power divided by the pulse repetition rate.

In all used processing configurations, a drill through with multi-pass ablation drilling was achieved. The number of required pulses to perforate the material and the processing time to treat the target area of $30 \times 5 \mathrm{~mm}^{2}(L \times W)$ vary significantly. From the ablation craters of a single pulse ablation of the different pulse energies, the spot size radius was experimentally determined to $51.6 \pm 2.6 \mu \mathrm{m}$.

A couple of microscope images from the bore hole entrance and exit under various conditions are shown in Fig. 2. 

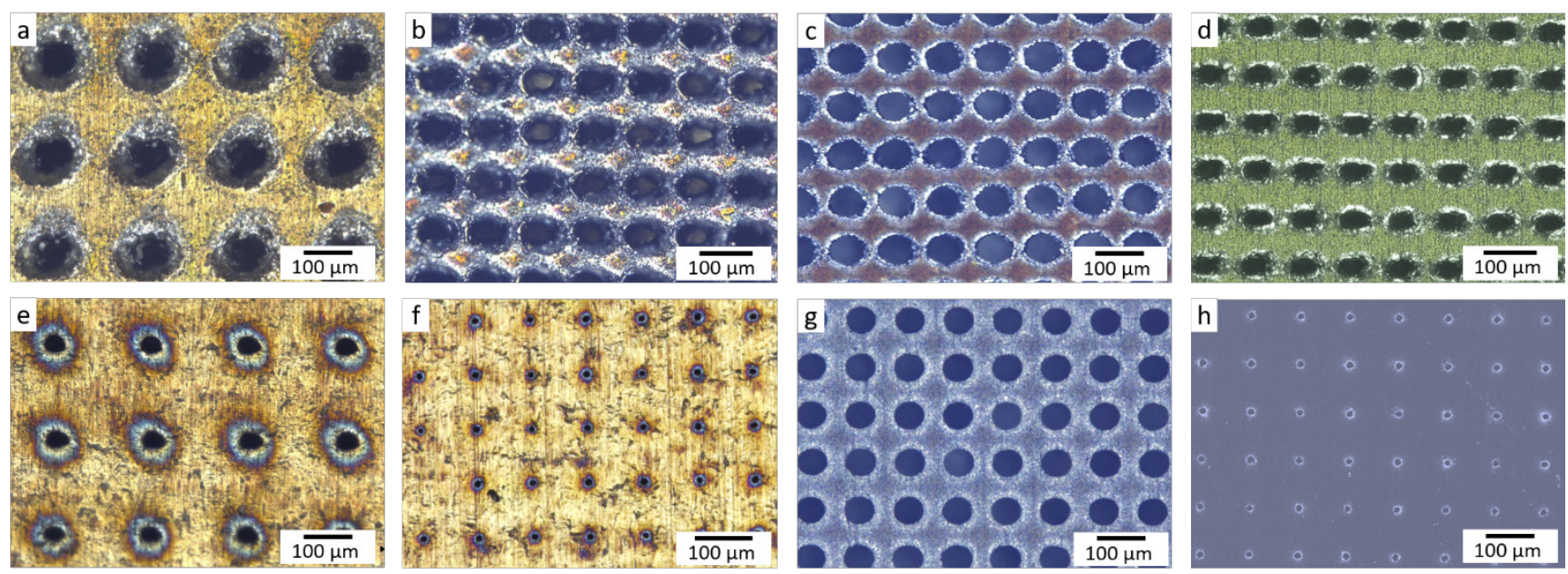

Fig. 2 Bore hole diameters on the beam entrance side (a-d) and the correlating exit side (e-h). (a/e) Stainless-steel drilled with 90 pulses of $240 \mathrm{~ns}$ duration and $1 \mathrm{~mJ}$ pulse energy, (b/f) steel treated with 220 pulses of $30 \mathrm{~ns}$ duration and $0.25 \mathrm{~mJ}$ pulse energy, (c/g) silicon wafer treated with 30 pulses of $1 \mathrm{~mJ}$ pulse energy and a pulse duration of $240 \mathrm{~ns}$ and $(\mathrm{d} / \mathrm{h})$ silicon processed with 60 pulses of $60 \mathrm{~ns}$ with an energy of $0.25 \mathrm{~mJ}$.

The entrance side is visible in the upper row, while the corresponding exit side is shown below.

All cases show the minimum required number of pulses to obtain a drill through state. Fig. $2(\mathrm{a} / \mathrm{e})$ show a stainlesssteel sample of $200 \mu \mathrm{m}$ thickness treated with 90 pulses of $23.9 \mathrm{~J} / \mathrm{cm}^{2}$ and a pulse duration of $240 \mathrm{~ns}$. The measured diameters are $122.2 \pm 5.6 \mu \mathrm{m}$ at the entrance and $20.3 \pm 3.7 \mu \mathrm{m}$ at the exit side. The spacing of holes is $200 \mu \mathrm{m}$, otherwise they would merge with each other at the entrance side. In Fig. 2 (b/f), steel is treated with 220 pulses of $30 \mathrm{~ns}$ pulse duration and a peak fluence of $6.0 \mathrm{~J} / \mathrm{cm}^{2}$. The spacing is $100 \mu \mathrm{m}$. The measured diameters are $70.5 \pm 2.3 \mu \mathrm{m}$ and $11.01 \pm 1.2 \mu \mathrm{m}$ at front and back side, respectively. In Fig. 2 (c/g), silicon was drilled with a peak fluence of $23.9 \mathrm{~J} / \mathrm{cm}^{2}$ and a pulse duration of $240 \mathrm{~ns}$. 30 pulses are required in this configuration to drill through the $180 \mu \mathrm{m}$ thick material. The spacing is $100 \mu \mathrm{m}$. This is the highest fluence used in the experiments, resulting in large diameters in front and back side of $61.8 \pm 1.7 \mu \mathrm{m}$ and $43.4 \pm 1.5 \mu \mathrm{m}$, respectively. The last example is shown in Fig. $2(\mathrm{~d} / \mathrm{h})$, a silicon wafer was treated with 60 pulses of $6.0 \mathrm{~J} / \mathrm{cm}^{2}$ at a pulse duration of $60 \mathrm{~ns}$. The diameters at entrance and exit side are $66.6 \pm 2.9 \mu \mathrm{m}$ and $12.9 \pm 0.9 \mu \mathrm{m}$, respectively.

The distribution of laser pulses in the multi-pass ablation has a significantly influence on the whole process, since a bore hole is hit with a frequency much lower, than the laser pulse repetition rate. Consecutive pulses hit the target along a scanned line and the process is continued in the next line. The number of lines is depending on the spacing of lines, called hatch $h$, and the width of the treated area $W$. With every repetition of the scan, the number of pulses per hole is increased by one. During the steel treatment up to 220 scans must be performed to obtain a drill through. No ablation besides a bore hole is visible from Fig. 2 (b/f), meaning an accurate pulse synchronization of all facets and over the whole processing time. The effective pulse frequency $f_{\text {eff }}$ is decoupled from the laser pulse repetition rate. It can be calculated following equation (1) and depends on the number of lines and the line rate of the polygon scanner $f_{\text {line }}$.

$$
f_{\text {eff }}=\frac{f_{\text {line }}}{\left(\frac{W}{h}+1\right)}
$$

Drilling a $5 \mathrm{~mm}$ width area with a spacing of $100 \mu \mathrm{m}$ in both directions with a laser frequency of $4 \mathrm{MHz}$ results in an effective pulse frequency per bore hole of only $11.88 \mathrm{~Hz}$. Using a $1 \mathrm{MHz}$ frequency instead would result in only $2.97 \mathrm{~Hz}$. Nevertheless, the reduced effective pulse frequency overcomes typical limitations of high frequency pulsed laser treatments such as heat accumulation and particle shielding between consecutive pulses.

For the application of a drilled material, especially as filter or micro nozzle, the hole geometry is an important value. From the analysis in the microscope the diameters of the drilled holes can be measured on the front and back side. Fig. 3 shows the evolution of the diameters with the applied number of pulses per position.

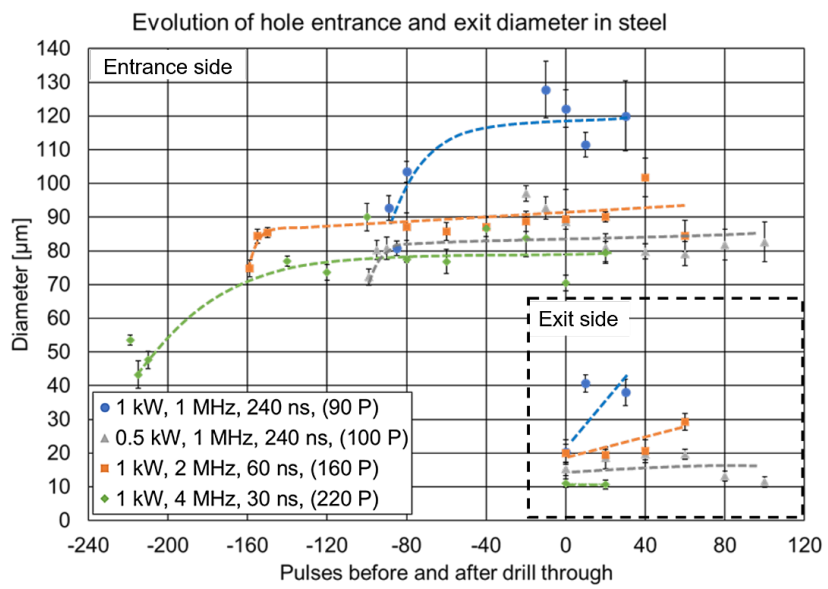

Fig. 3 Evolution of hole entrance and exit diameter on stainlesssteel over the number of pulses before $(<0)$ and after $(>0)$ drill through. The required number of pulses to drill through is set to 0 for all cases. The lines are drawn to guide the eyes.

Due to the fast scan speed, a relevant distance is traveled in the scanning direction during the pulse duration. As a consequence, the hole geometry is prolonged in scanning direction. Further investigations are done with the diameter perpendicular to the scanning direction.

Since the number of pulses to drill through differs with the processing conditions, the abscissa was set to zero at the 
drill through number of pulses. The absolute value is given in brackets behind the processing conditions. Thus, the development of entrance and exit diameters is shown also for additionally applied pulses $\geq 0$. On the contrary, the diagram shows negative number of pulses, meaning pulses before drilled through. In this state $(<0)$ the treated position contains blind holes with an entrance diameter only.

From the diagram it is visible, that the entrance diameter increases in the beginning fast with the number of pulses and reaches then an almost constant state with very slow increase. Processing with $4 \mathrm{MHz} @ 30 \mathrm{~ns}$, the diameter increases from around $50 \mu \mathrm{m}$ towards approximately $80 \mu \mathrm{m}$ within 80 pulses and stays on this size without further increase. 220 pulses are required to drill through. Processing with $2 \mathrm{MHz}$ (a) $60 \mathrm{~ns}$ lead to diameters of around $90 \mu \mathrm{m}$ after 80 pulses and requires 160 pulses to drill through. For $1 \mathrm{MHz}$ (a) 240 ns, the largest diameters are observed with 120 to $130 \mu \mathrm{m}$. Drilling through requires 90 pulses. For comparison, an experiment was performed at $1 \mathrm{MHz}$ @240 ns with only the half available power of $500 \mathrm{~W}$. In this case, the diameter stays around $80 \mu \mathrm{m}$ and 100 pulses are required to drill through.

The size of the entrance diameters increases with the pulse energy and the pulse duration. This behavior is expectable, since the pulse energy is Gaussian distributed within the focal spot. Consequently, the diameter of the laser fluence exceeding the threshold fluence increases with the pulse energy. Furthermore, the thermal diffusion increases with the pulse duration melting material in larger distances from the spot center.

At the exit side, a similar behavior of increasing diameters with pulse energy and pulse duration can be observed. $4 \mathrm{MHz}$ @30ns treatment achieve an initial diameter of $11.0 \pm 1.2 \mu \mathrm{m}$ at the exit. After additional 20 pulses the diameter is not changed and determined to $10.7 \pm 1.3 \mu \mathrm{m}$. Treating with $2 \mathrm{MHz} @ 60$ ns leads to an initial exit diameter of $20.0 \pm 2.8 \mu \mathrm{m}$. With further 60 pulses, an increase to $29.2 \pm 2.5 \mu \mathrm{m}$ was observed. The highest pulse energy configuration of $1 \mathrm{MHz} @ 240$ ns leads to $20.3 \pm 3.7 \mu \mathrm{m}$ diameters at the first observed drill through. The outlet diameters increase strongly towards $38.0 \pm 3.9 \mu \mathrm{m}$ after 30 additional pulses. The $500 \mathrm{~W}$ treatment with $1 \mathrm{MHz} @ 240$ ns leads to diameters between 15 and $20 \mu \mathrm{m}$ from the initial drill through up to 100 additional pulses. Consequently, the exit diameters reach a size of 15 to $30 \%$ of the entrance diameters, whereby this ratio increase with the pulse duration and pulse energy.

A similar diagram of the entrance and exit diameters for the silicon wafers is shown in Fig. 4. Again, the pulse number of the initial drilling through is set to zero, to compare the diameter evolution before and after the perforation.

The diameters at the entrance side increase with the number of pulses in the beginning strongly, then slower. Compared to the steel samples, the number of applied pulses is in general lower. Thus, the saturation of the entrance diameter is maybe not reached within the performed tests and an increasing diameter is observed after drilling through.

A relatively high number of pulses is applied in the case of $4 \mathrm{MHz}$ @30 ns processing performed again with the maximum power of $1 \mathrm{~kW}$. The diameter after a single pulse is measured to be $26.9 \pm 1.7 \mu \mathrm{m}$ it increases to $44.7 \pm 2.3 \mu \mathrm{m}$ after 20 pulses and stays in this range up to

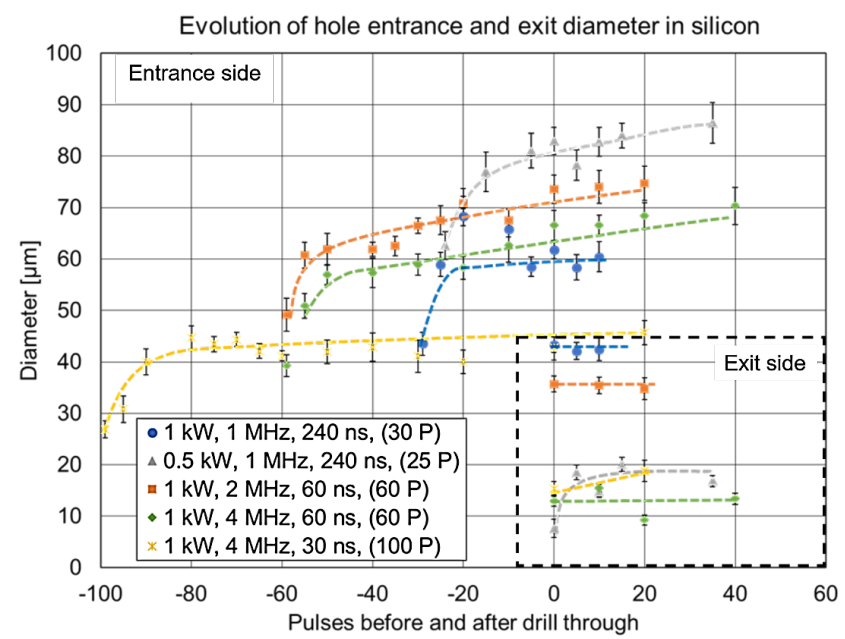

Fig. 4 Evolution of hole entrance and exit diameter of silicon wafers over the number of pulses before $(<0)$ and after $(>0)$ drill through. The lines are drawn to guide the eyes.

120 pulses $(45.7 \pm 2.3 \mu \mathrm{m})$. A drilling through is achieved after 100 pulses. A treatment with $4 \mathrm{MHz}$ but a doubled pulse duration of $60 \mathrm{~ns}$ causes enlarged diameters starting from $29.3 \pm 2.1 \mu \mathrm{m}$ increasing quickly to $56.9 \pm 2.9 \mu \mathrm{m}$ after 10 pulses and increases further. Thus, the diameter at the perforation is measured to be $66.6 \pm 2.9 \mu \mathrm{m}$ after 60 pulses, while 40 additional pulses increase the entrance diameter to $70.4 \pm 3.6 \mu \mathrm{m}$ (after 100 in total). Using $60 \mathrm{~ns}$ pulses at a repetition rate of $2 \mathrm{MHz}$ increases the diameters further. After a single pulse, a diameter of $49.2 \pm 3.2 \mu \mathrm{m}$ can be observed. It increases strongly towards $62.0 \pm 2.5 \mu \mathrm{m}$ after 10 pulses and then slightly. The perforation is obtained after 60 pulses, where the entrance diameter is measured to be $73.6 \pm 2.8 \mu \mathrm{m}$. Additional 20 pulses increase the diameter to $74.7 \pm 3.3 \mu \mathrm{m}$ ( 80 pulses in total). Using the highest pulse energy with $1 \mathrm{MHz} @ 240$ ns results in smaller diameters. The single pulse crater has a dimeter of $43.5 \pm 2.2 \mu \mathrm{m}$ but after 5 pulses the diameters stays between 56.6 and $70.2 \mu \mathrm{m}$ without increasing trend. The material is drilled through after 30 pulses. Again a $500 \mathrm{~W}$ experiment was performed with $1 \mathrm{MHz} @ 240$ ns. In this case, the diameter starts from $62.6 \pm 2.7 \mu \mathrm{m}$ and increases to $77.0 \pm 3.9 \mu \mathrm{m}$ after 10 pulses and further towards $86.5 \pm 4.0 \mu \mathrm{m}$ after 60 pulses. The perforation is reached after 25 pulses.

The comparison of the $30 \mathrm{~ns}$ and $60 \mathrm{~ns}$ with $0.25 \mathrm{~mJ}$ pulse energy obtained from $4 \mathrm{MHz}$ pulse repetition rate show clearly, that the pulse duration has an influence on the diameter. The values of the $60 \mathrm{~ns}$ pulses are approximately 1.5 times larger than in the case of $30 \mathrm{~ns}$ pulses. The thermal diffusion length correlating with $\sqrt{\tau}$ is increased with the pulse duration and consequently also the ablated area [17].

In the experiments the pulse duration is doubled and the thermal diffusion length should be increased by $\sqrt{2} \approx 1.41$, which corresponds to the observed results. Also, the increase of diameters between the $60 \mathrm{~ns}$ pulses at $2 \mathrm{MHz}$ and the $500 \mathrm{~W}, 1 \mathrm{MHz} @ 240 \mathrm{~ns}$ with $0.5 \mathrm{~mJ}$ pulse energy in both cases, can be declared with the enlarged heat diffusion depending on the pulse duration.

The increase of diameters between $60 \mathrm{~ns}$ pulses of $0.25 \mathrm{~mJ}$ and $0.50 \mathrm{~mJ}$ pulse energy can be declared with the energy distribution within a Gaussian beam, where the laser 

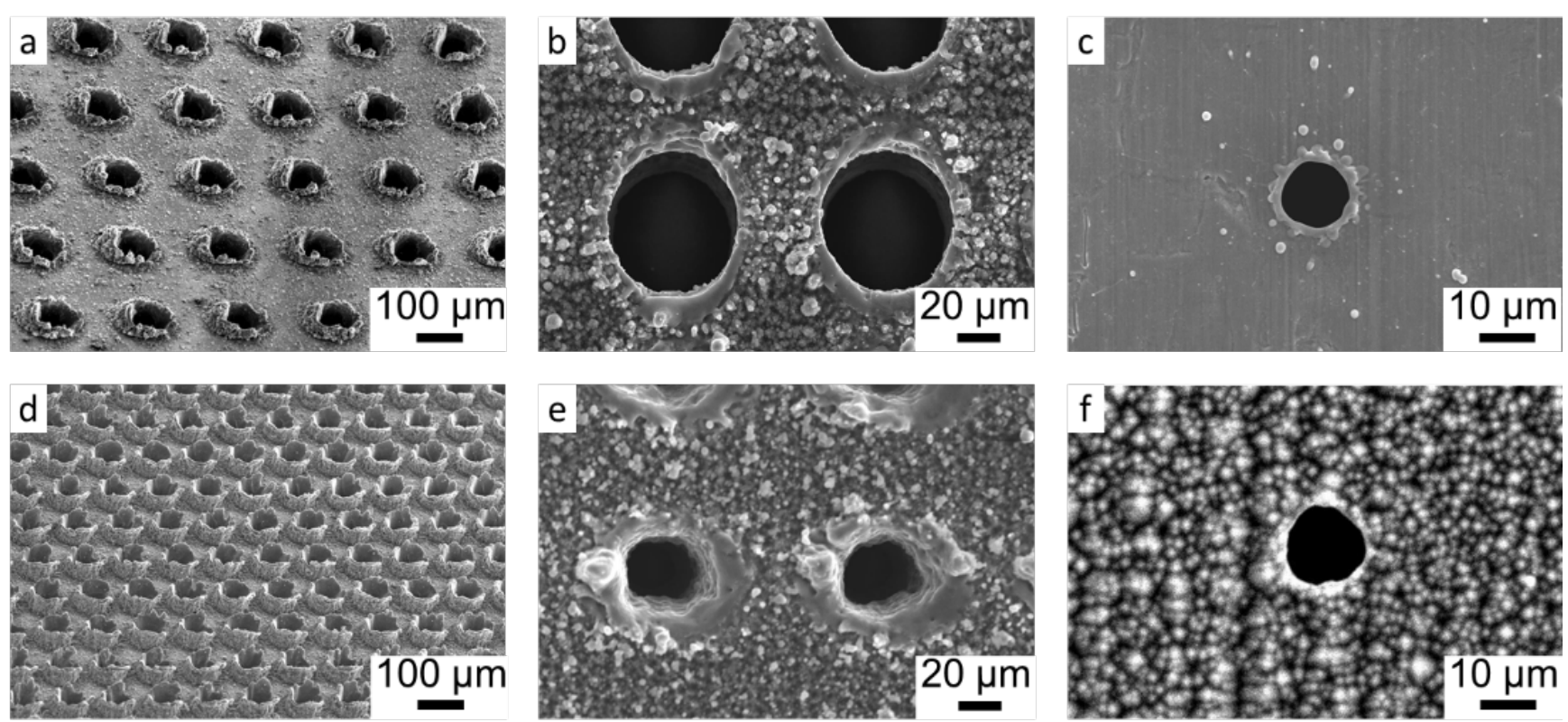

Fig. 5 SEM images of the entrance side of (a) steel after 90 pulses and (b) silicon after 30 pulses treated with $240 \mathrm{~ns}$ and 1 mJ. (c) Exit and (d) entrance of steel after 220 pulses of $30 \mathrm{~ns}$ and $0.25 \mathrm{~mJ}$, (e) entrance of silicon after 100 pulses treated with $30 \mathrm{~ns}$ and $0.25 \mathrm{~mJ}$ and (f) exit side of silicon after 60 pulses of $60 \mathrm{~ns}$ and $0.25 \mathrm{~mJ}$.

fluence exceeds the threshold fluence in an enlarged diameter with increased pulse energy.

That the diameters of the $1 \mathrm{~kW}$ experiments at $240 \mathrm{~ns}$ pulses with $1 \mathrm{MHz}$ respectively $1 \mathrm{~mJ}$ pulse energy are smaller compared to the $500 \mathrm{~W}$ at the same pulse duration as well as the $60 \mathrm{~ns}$ experiments is not really clear. A possible explanation is, that the very high pulse energy, and consequently a very high peak fluence, vaporizes the material in the center of the irradiated area. Thus, the energy is not available for heat diffusion in neighbored less or not irradiated areas, since it is firstly used to overcome the vaporization enthalpy and secondly, the material is removed into the ambient as gas or plasma still carrying the absorbed laser energy [18]. Furthermore, higher pulse energy and longer pulses will lead to increased melt ejection, removing absorbed energy and reduces the heat dissipation.

The exit side diameters show a different order. The lowest diameters were observed at the $500 \mathrm{~W}$ tests with $1 \mathrm{MHz}$ (a) $240 \mathrm{~ns}$. At the initial drill through, diameters of $7.6 \pm 1.8 \mu \mathrm{m}$ can be measured. From 5 to 35 additional pulses, values from $14.7 \mu$ to $20 \mu \mathrm{m}$ can be measured, which corresponds to only $20 \%$ of the entrance diameter. An increasing trend is not visible. The full power experiments with $4 \mathrm{MHz} @ 30 \mathrm{~ns}$ lead to $15.4 \pm 1.3 \mu \mathrm{m}$ diameters increasing slightly to $18.8 \pm 2.1 \mu \mathrm{m}$ after 20 additional pulses. The same pulse energy at $60 \mathrm{~ns}$ and $4 \mathrm{MHz}$ lead to values between $9 \mu \mathrm{m}$ and $16 \mu \mathrm{m}$ independently from the number of additional pulses after drill through. Significantly larger diameters were observed from the higher fluence tests performed with $2 \mathrm{MHz} @ 60$ ns. The initial drill through value is measured to be $35.7 \pm 1.3 \mu \mathrm{m}$. No increase visible up to 20 additional pulses. The largest diameters are measured from the $1 \mathrm{MHz} @ 240$ ns pulses with the full power of $1 \mathrm{~kW}$. From the initial perforation up to 10 additional pulses, values of $43.4 \pm 2.2 \mu \mathrm{m}$ can be measured. In the $1 \mathrm{~mJ}$ experiments, the exit diameter amounts more than $70 \%$ on the entrance diameter.
The exit diameters increase with the pulse energy. From the experiments with the same pulse energy at different pulse durations, it is also visible, that shorter pulse durations result in larger exit diameters. This can be explained, since the heat dissipation is reduced and more energy is available for ablation and melt ejection in the absorption zone. Thus, a less tapered volume is removed per pulse, resulting in less tapered holes compared to longer pulses with more lateral heat dissipation. Consequently, also the exit to entrance ratio of the diameters increases with higher pulse energy, and shorter pulse durations.

Fig. 5 shows some bore hole entrance and exit images obtained from scanning electron microscopy (SEM).

It is visible in Fig. $5(\mathrm{a} / \mathrm{d})$, that burr is formed on the steel samples on the entrance side. Thereby, the height of the burr increases with the number of pulses, since the ejected melt has enough time to solidify until the next laser pulse hits the hole. Consequently, with reduced pulse energy and more pulses the burr raises even the pulse duration becomes smaller. In the case of silicon (Fig. $5 \mathrm{~b} / \mathrm{e}$ ), barely burr or melt is visible. Also, the back sides of both steel and silicon show no significant burr formation, as visible in Fig. 5 (c/f), respectively.

For high throughput treatments, it is also interesting, which time is required to run a process. Table 1 summarize the process conditions and the required number of pulses to drill through $N_{P}$. Additionally, the processing time $t_{p r o c}$ is listed for all used configurations.

The number of required pulses to drill through varies with the condition between 90 and 220 on steel samples and between 25 and 100 on silicon.

On both materials a trend is visible, that the number of required pulses increase with decreasing pulse energy and pulse duration. The influence of the fluence follows usually the logarithmic correlation between fluence $F$ and ablation depth $d: d=1 / \alpha \cdot \ln \left(F / F_{t h}\right)$, with the material specific absorption coefficient $\alpha$ and threshold fluence $F_{t h}$. Consequently, a decrease of the fluence, results in a reduced 
ablation depth per pulse, and more pulses are needed to ablate a certain depth.

It is also visible, that treatments with the same pulse energy at different pulse durations results in a higher number of required pulses on shorter pulse durations. A possible explanation is, that the increased pulse peak power is increasing the amount of vaporized material during shorter laser pulses. Thus, vaporization is reached faster, reducing the heat conduction from the hot center of the absorption zone towards less or not irradiated areas. Furthermore, the absorption coefficient is temperature depending. Especially in silicon it is increasing strongly with the temperature [19].

Table 1 Required number of laser pulses to drill through and corresponding processing time for the target area under various processing conditions.

\begin{tabular}{ccccccc}
\hline Material & $\begin{array}{c}E_{P} \\
{[\mathrm{~mJ}]}\end{array}$ & $\begin{array}{c}f_{P R R} \\
{[\mathrm{MHz}]}\end{array}$ & $\begin{array}{c}\tau \\
{[\mathrm{ns}]}\end{array}$ & $\begin{array}{c}v_{\text {scan }} \\
{[\mathrm{m} / \mathrm{s}]}\end{array}$ & $\begin{array}{c}N_{P} \\
{[-]}\end{array}$ & $\begin{array}{c}t_{\text {proc }} \\
{[\mathrm{s}]}\end{array}$ \\
\hline Steel & 1.00 & 1 & 240 & 200 & 90 & 7.71 \\
Steel & 0.50 & 1 & 240 & 200 & 100 & 8.57 \\
Steel & 0.50 & 2 & 60 & 400 & 160 & 6.99 \\
Steel & 0.25 & 4 & 30 & 400 & 220 & 18.50 \\
Silicon & 1.00 & 1 & 240 & 100 & 30 & 10.20 \\
Silicon & 0.50 & 1 & 240 & 100 & 25 & 8.41 \\
Silicon & 0.50 & 2 & 60 & 200 & 60 & 10.1 \\
Silicon & 0.25 & 4 & 60 & 400 & 60 & 2.57 \\
Silicon & 0.25 & 4 & 30 & 400 & 100 & 8.51 \\
\hline
\end{tabular}

Thus, higher pulse energies and shorter pulse durations reduce the penetration depths more than longer pulses or less pulse energy. That in the case of silicon, the $500 \mathrm{~W}$ experiment drills through faster, than the $1 \mathrm{~kW}$ trials indicate, that the optimal allocation of laser fluence and pulse duration is not found with the used configurations. Further investigations would be needed to optimize this laser matter interaction. However, the maximum laser power is only available in defined combinations of pulse energy and pulse durations. A processing with less than the maximum power is not sufficient to the investment costs. Furthermore, the value of pulses to reach a perforation has no sufficient character to estimate the drilling rate (number of holes / time), which is also depending on the laser pulse repetition rate.

The observed processing time also varies strongly between $7.71 \mathrm{~s}$ and $18.50 \mathrm{~s}$ on the stainless-steel and $2.57 \mathrm{~s}$ and $10.20 \mathrm{~s}$ on the silicon samples. Since it increases on the one hand with the number of pulses to drill through but on the other hand also with the total number of drilled holes in the target area, it can be used to determine the drilling rate. Therefore, the whole number of drilled holes, must be taken into account depending on the hole spacing.

The scan speed $v_{\text {scan }}$ and the laser pulse repetition rate $f_{P R R}$ determine the distance of two consecutive emitted laser pulses during the distributed on the surface. This pulse to pulse distance $P D$ follows $P D=v_{s c a n} / f_{P R R}$. The scan speed correlates to the pulse frequency to obtain a $100 \mu \mathrm{m}$ or $200 \mu \mathrm{m}$ spacing. The hatch is set in the experiments similar to the pulse to pulse distance along the scanning direction, in order to obtain a rectangular arrangement of holes. Hence, the drilling rate $R_{\text {Drill }}$ can be calculated with equation (2) from the total number of drilled holes and the processing time, where $L$ describes the length of the treated area in scanning direction.

$$
R_{\text {Drill }}=\left(\frac{W}{h}+1\right) \cdot\left(\frac{L}{P D}+1\right) \cdot \frac{1}{t_{\text {proc }}}
$$

The resulting drilling rates are shown in Fig. 6 together with the used processing parameters.

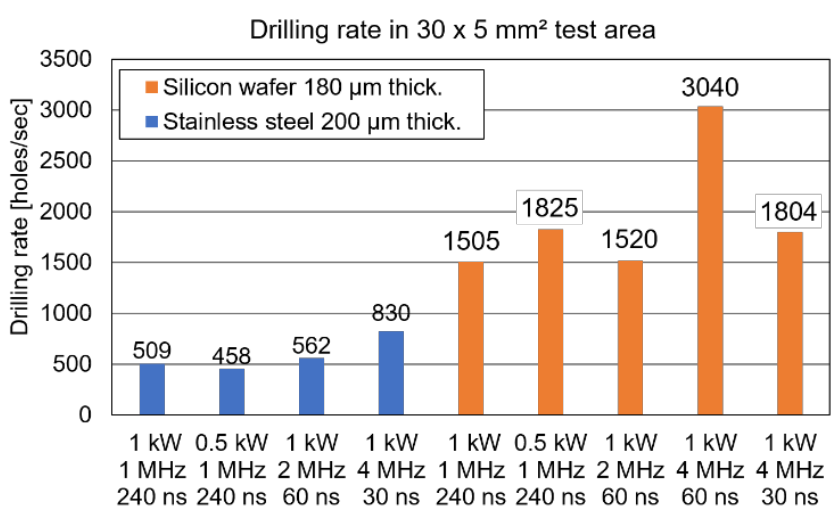

Fig. 6 Achieved drilling rate of $200 \mu \mathrm{m}$ thick stainless-steel and $180 \mu \mathrm{m}$ thick silicon samples under various processing conditions.

First of all, it is visible, that the drilling rate of steel is much lower than of silicon for all cases, which is caused by the different laser matter interaction of both materials. Within the stainless-steel samples, the drilling rate increases with the laser pulse repetition rate using $1 \mathrm{~kW}$ average laser power from 509 holes/s drilled with $1 \mathrm{MHz} @ 240$ ns pulses via 562 holes/s at $2 \mathrm{MHz} @ 60$ ns pulses to 830 holes/s using $4 \mathrm{MHz} @ 30$ ns pulses. The experiment with only half power of $500 \mathrm{~W}$ at $1 \mathrm{MHz}$ and $240 \mathrm{~ns}$ pulse duration leads to a reduced drilling rate of 458 holes/second.

In the case of silicon, the drilling rate of the $1 \mathrm{MHz}$ @ 240 ns and $2 \mathrm{MHz} @ 60$ ns are the lowest observed with 1505 holes/s and 1520 holes/s, respectively. The $4 \mathrm{MHz} @$ $30 \mathrm{~ns}$ has little bit higher drilling rate of 1804 holes/s. A similar value is obtained for the $500 \mathrm{~W}, 1 \mathrm{MHz} @ 240 \mathrm{~ns}$ process. The significantly highest drilling rate of 3040 holes/s is achieved under $4 \mathrm{MHz} @ 60$ ns processing. The increase of drilling rate with the pulse frequency is here not as clear as in the steel samples, since the laser matter interaction is very different in the used pulse conditions. Consequently, the higher number of pulses to perforate the material is counteracting the higher pulse frequency. Nevertheless, a $60 \mathrm{~ns}$ pulse treatment with $4 \mathrm{MHz}$ and a pulse energy of $0.25 \mathrm{~mJ}$ results in a very high drilling rate.

The drilling rate increases with the laser pulse repetition rate as well as with the effective pulse frequency. As a result, the total process time can be determined from both, the number of pulses to drill through, and the effective pulse frequency: $t_{\text {proc }}=N_{p} / f_{\text {eff }}$. Hence, the drilling rate can be estimated from the set processing parameters. The pulse separation depends on the laser pulse repetition rate and the scan speed, while the ratio of line frequency to scan speed is defined by the used optical configuration. It can be described as $C_{f v}$ following $C_{f v}=f_{\text {line }} / v_{\text {scan }}$. In the used set up, $C_{f v}$ is approximately 1.5 . The drilling rate can be described in this way either with the line rate and the pulse distance or with the pulse repetition rate and the scan speed in equation (3).

$$
R_{\text {drill }}=\frac{f_{\text {line }}}{N_{p}}\left(\frac{L}{P D}+1\right)=\frac{C_{f v} \cdot f_{P R R} \cdot L+C_{f v} \cdot v_{\text {scan }}}{N_{P}}
$$



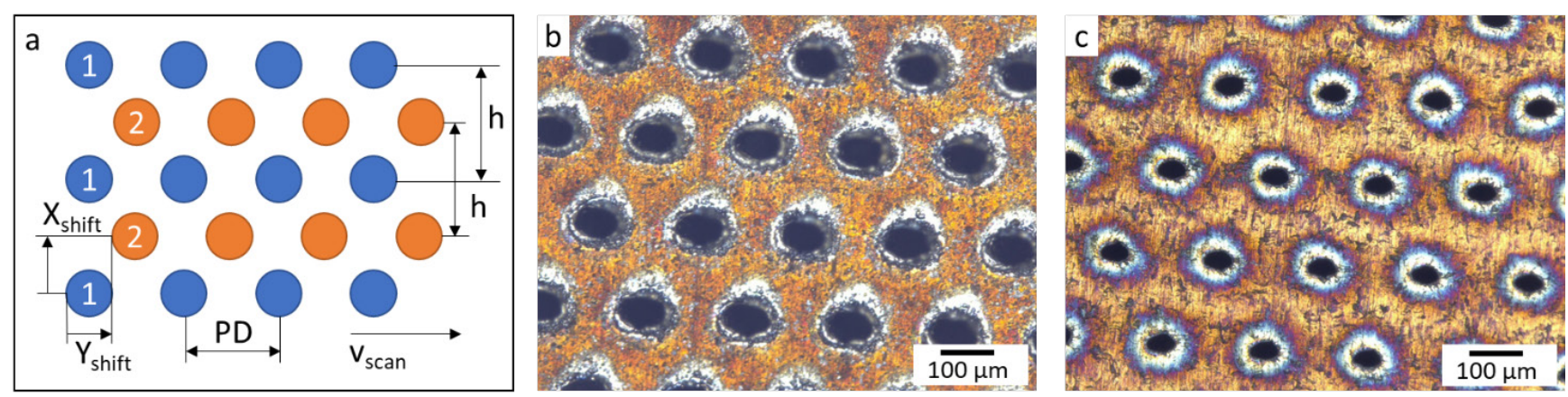

Fig. 7 Using the integrated shifter function of the polygon scanner allows non-rectangular bore hole arrangements for example hexagonal arrays with a $15 \%$ higher hole density. (a) Processing strategy with enlarged rectangular array (blue, 1) and X and Y shifted intermediate array (orange, 2). (b) Resulting bore holes at the entrance side with $103.6 \pm 7.4 \mu \mathrm{m}$ diameters and (c) the exit side with $32.9 \pm 1.2 \mu \mathrm{m}$ processed with 240 pulses of $1 \mathrm{~mJ}$ pulse energy and a pulse duration of $240 \mathrm{~ns}$.

The equation shows the mathematical correlation between the drilling rate and the pulse repetition rate, as well as the deflection speed of the scanner. Another value in this equation is the length of the treated area $L$. In the experiments it is only $30 \mathrm{~mm}$. An increase would lead nearly to a proportional higher drilling rate without increasing the processing time. Of course, the maximum length is limited by the used focusing objective to $300 \mathrm{~mm}$ and optical distortions at the edges of the scan field can shrink the useable length further. Assuming an elongated target area without optical distortions of $153 \mathrm{~mm}$ (6 inch), would increase the drilling rate by factor 5 . The maximum archived drilling rate on silicon of 3040 holes/s can be increased in this way to $15,400 \mathrm{holes} / \mathrm{s}$, using exactly the same laser and scanner process $4 \mathrm{MHz} @ 60 \mathrm{~ns}$ with $400 \mathrm{~m} / \mathrm{s}$ scan speed. It would just use the scan field more efficient with line efficiency of above $50 \%$. This results from the working principle of polygon mirror scanners. The rotating mirror scans always the whole length of the scan field, just turning the laser off outside the working area. Further increase towards 30,000 holes/s can be achieved using larger scan length towards $300 \mathrm{~mm}$ accepting possibly deviations at the scan field edges.

Smaller targets can be treated more properly with smaller objectives, respectively smaller focal length and scan field size, which increase the line efficiency. This would increase also line frequency to scan speed ratio $C_{f v}$ since the scan speed scales with the focal length.

Finally, a variation of the pulse distribution can increase the hole density. Fig. 7 shows hexagonal arranged pulses. In this procedure, every second line is intended, which is achieved from two rectangular arranged patterns as visible in Fig. 7(a). Firstly, the hatch distance is increased to the distance of $1.732 P D$, meaning two lines in hexagonal pattern, while the pulse to pulse distance is kept constant. The first rectangular pattern is processed. Afterwards, the internal shifter function of the scanner rearranges the rectangular pattern with an offset in $\mathrm{x}$ and $\mathrm{y}$ direction, where $\mathrm{x}$ is shifted by the half hatch $(0.866 P D)$ and $y$ is intended by a half of the pulse to pulse distance $(0.5 P D)$. Then the second rectangle is processed. At the end a hexagonal pattern with the target distance $P D$ is obtained at the front (Fig. 7(b)) and back (Fig. 7(c)) side of the material. The hole density is increased by $15.4 \%$ compared to a rectangular arrangement of the same hole spacing. The processing time, is increased similarly.

\section{Conclusions}

A polygon mirror scanner has been used in multi-pass ablation processing to drill holes in $200 \mu \mathrm{m}$ thick stainlesssteel and $180 \mu \mathrm{m}$ thick silicon wafers. The experiments were performed with a $1 \mathrm{~kW}$ average power nanosecond pulsed fiber laser with $1064 \mathrm{~nm}$ wavelength. Thereby, the maximum available laser power can be obtained with different pulse repetition rates from 1 to $4 \mathrm{MHz}$ with respectively decreasing pulse energy from 1 to $0.25 \mathrm{~mJ}$. Furthermore, the repetition rates are coupled to different pulse durations between 240 and $30 \mathrm{~ns}$.

The distribution of consecutive laser pulses along the whole treated area decouples the laser pulse repetition from the effective pulse frequency at a certain bore hole. Thus, typical limitations of high repetition rate pulsed laser treatments as heat accumulation and shielding effects are strongly reduced. This kind of processing requires high scan speeds, since the spacing of the holes depends directly on the speed and the pulse repetition rate. A $100 \mu \mathrm{m}$ spacing at $4 \mathrm{MHz}$ is only possible with $400 \mathrm{~m} / \mathrm{s} \mathrm{scan}$ speed. At the same time, the accuracy must be high enough to hit the same position in every repetition of the scan. In polygon mirror scanning, this is only possible with pulse synchronization. Here, the laser is possible to adjust the pulse repetition rate to the position frequency of the scanner. In this way, the laser frequency is synchronized to the rotation frequency of the polygon wheel and the holes are hit properly.

Besides the fluence between the different processing conditions, also the pulse peak power changes, resulting in a different laser matter interaction. Hence, a different number of laser pulses is required to achieve a through hole and the bore hole geometry varies also. The entrance and exit diameters were measured for all experiments, showing ratios at the exit between 20 and $70 \%$ of the entrance diameter. Depending on the perforation pulse number and the pulse repetition rate, the drilling rate can be determined for the machined area. An estimation for a more efficient use of the scan field can be done in addition. On steel, the highest drilling rate was measured to be 830 holes/s using $30 \mathrm{~ns}$ pulsed at $4 \mathrm{MHz}$ in a $30 \times 5 \mathrm{~mm}$ area. Increasing the machined length towards $150 \mathrm{~mm}$ would increase the drilling rate to 4,100 holes/s without any changes in the processing parameters. This is an effect of the working principle of polygon scanners, which scans always the whole length of the scan field, just turning the laser off in areas outside the target. The maximum obtained drilling rate of silicon using $60 \mathrm{~ns}$ pulses 
at $4 \mathrm{MHz}$ was determined to $3,040 \mathrm{holes} / \mathrm{s}$ and can be increased to 15,150 holes/s under optimized scan field utilization. Further increase of the machined area towards the full scan length of $300 \mathrm{~mm}$ would increase the drilling rate further up to 30,000 holes/s at the full size along the fast scanning axis.

At the end, a processing strategy was shown to obtain hexagonal arranged holes from two rectangular arrays. This is increasing the hole density to $115 \%$ compared to rectangular arranged holes.

\section{References}

[1] R. Weber, T. Graf, P. Berger, V. Onuseit, M. Wiedenmann, C. Freitag, and A. Feuer: Opt. Express, 22, (2014) 11312.

[2] A. Ancona, F. Röser, K. Rademaker, J. Limpert, S. Nolte, and A. Tünnermann: Opt. Express, 16, (2008) 8958.

[3] A. Ancona, S. Döring, C. Jauregui, F. Röser, J. Limpert, S. Nolte, and A. Tünnermann: Opt. Lett., 34, (2009) 3304.

[4] J. Finger and M. Reininghaus: Opt. Express, 22, (2014) 18790.

[5] U. Löschner, J. Schille, F. Ullmann, A. Streek, L. Hartwig, and S. Klötzer: Proc. 24th Int. Scientific Conference, Mittweida, Germany, (2015) p. 70.

[6] R. D. Loor, L. Penning, and R. Slagle: Laser Tech. J., 11, (2014) 32.

[7] K. van der Straeten, O. Nottrodt, M. Zuric, A. Olowinsky, P. Abels, and A. Gillner: Proc. 10th CIRP Conference on Photonic Technologies, Fürth, Germany, (2018) p. 491.

[8] A. Streek and M. Lee: Laser Tech. J.,14, (2017) 22.

[9] U. Dürr: Laser Tech. J., 5, (2008) 57.

[10] V. Tomkus, V. Girdauskas, J. Dudutis, P. Gečys, V. Stankevič, and G. Račiukaitis: Opt. Express, 26, (2018) 27965.

[11] T. N. Kim, K. Campbell, A. Groisman, D. Kleinfeld, and C. B. Schaffer: Appl. Phys. Lett., 86, (2005) 201106

[12] K. Ogawa, H. Nakagawa, F. Murase, and S. Nishida: Key Eng. Mater., 516, (2012) 54.

[13] K. Rahim and A. Mian: J Electron. Packag., 139, (2017) 030801 .

[14] J. M. Gee, W. K. Schubert, and P. A. Basore: Proc. 23rd IEEE Photovoltaic Specialists Conference, Louisville (KY), USA, (1993) p. 265.

[15] M. Antar, D. Chantzis, S. Marimuthu, and P. Hayward: Proc.18th CIRP Conference on Electro Physical and Chemical Machining, Tokyo, Japan, (2016) p. 526.

[16] A. Stephen, G. Schrauf, S. Mehrafsun, and F. Vollertsen: Proc. 5th Machining Innovations Conference, Garbsen, Germany, (2014) p. 130.

[17] P. Baeri and S. U. Campisano: "Laser Annealing of Semiconductors" ed. by J. M. Poate and J. W. Mayer (Academic Press, New York, 1982) p. 75.

[18] R. F. Wood and G. E. Giles: Phys. Rev. B, 23, (1981) 2923.

[19]E. Ohmura: "Heat Transfer - Engineering Applications" ed. by V. Vikhrenko, (InTechOpen, 2011).

(Received: June 23, 2020, Accepted: October 30, 2020) 